\section{OPEN ACCESS}

Edited by:

Sherven Sharma,

VA Greater Los Angeles Healthcare

System (NHA), USA

Reviewed by:

Mohey Eldin El Shikh,

Queen Mary University of London,

Manel Juan,

Hospital Clínic de Barcelona, Spain

${ }^{*}$ Correspondence:

Wei Xu

xuwei10000@hotmail.com

tThese authors have contributed equally to this work.

Specialty section:

This article was submitted to Cancer Immunity and Immunotherapy,

a section of the journal

Frontiers in Immunology

Received: 02 October 2016 Accepted: 15 December 2016

Published: 27 December 2016

Citation:

Sun D-P, Wang L, Ding C-Y, Liang J-H, Zhu H-Y, Wu Y-J, Fan L, Li J-Y and Xu W (2016) Investigating

Factors Associated with Thymic Regeneration after Chemotherapy in

Patients with Lymphoma.

Front. Immunol. 7:654.

doi: 10.3389/fimmu.2016.00654

\title{
Investigating Factors Associated with Thymic Regeneration after Chemotherapy in Patients with Lymphoma
}

Dao-Ping Sun ${ }^{1,2+}$, Li Wang ${ }^{1+}$, Chong-Yang Ding ${ }^{3}$, Jin-Hua Liang' ${ }^{1}$, Hua-Yuan Zhu', Yu-Jie Wu', Lei Fan', Jian-Yong $\mathrm{Li}^{1}$ and Wei $\mathrm{Xu}^{1 *}$

${ }^{1}$ Department of Hematology, The First Affiliated Hospital of Nanjing Medical University, Jiangsu Province Hospital, Collaborative Innovation Center for Cancer Personalized Medicine, Nanjing Medical University, Nanjing, China, ${ }^{2}$ Department of Hematology, Jining No.1 People's Hospital, Jining, China, ${ }^{3}$ Department of Nuclear Medicine, The First Affiliated Hospital of Nanjing Medical University, Jiangsu Province Hospital, Nanjing, China

The factors involved in thymus regeneration after chemotherapy has not been sufficiently explored. This study was aimed to identify the clinical characteristics and single-nucleotide polymorphisms in the gene (IL7R) encoding IL-7R $\alpha$ associated with thymus renewal after chemotherapy in Chinese Han individuals with lymphoma. The dynamics of thymic activity in 134 adults with Hodgkin lymphoma $(\mathrm{HL})$ and B cell lymphoma from baseline to 12 months post-chemotherapy were analyzed by assessing thymic structural changes using serial computed tomography scans and correlating these with measurements of thymic output by concurrent analysis of single-joint T-cell receptor excision circles (sjTREC) and CD31+ recent thymic emigrants (RTE) in peripheral blood. The association of clinical variables and $I L 7 R$ polymorphisms with the occurrence of rebound thymic hyperplasia $(\mathrm{TH})$ and the recovery of thymic output following chemotherapy were evaluated. Thymic regeneration was observed, with the evidence that TH occurred in 38/134 (28.4\%) cases, and thymic output, assessed by CD31+ RTE numbers and sjTREC content, recovered to baseline levels within 1 year after the end of therapy. The frequencies of the $T$ allele and $T T+$ GT genotype of rs7718919 located in the promoter of IL7R were significantly higher in patients with $\mathrm{TH}$ compared with those without $\mathrm{TH}(P=0.031$ and 0.027 , respectively). In contrast, no significant difference was found between two groups with respect to the distribution of allele and genotype frequencies of rs6897932. By general linear models repeated-measure analysis, rs7718919 and rs6897932 were determined to exert no significant effects on the recovery of thymic output after therapy. Univariate analysis revealed host age under 30 , the diagnosis of $\mathrm{HL}$, baseline thymic index and CD31+ RTE counts, and rs7718919 genotype as potential predictors for TH after chemotherapy $(P<0.05)$; after multivariate adjustment, only host age was independently associated with the occurrence of $\mathrm{TH}$ (odds ratios $=4.710,95 \%$ confidence intervals: $1.727-12.845, P=0.002)$. These findings indicate that patient age is an independent predictor for thymic regrowth after chemotherapy, which should promote awareness among physicians to make a timely diagnosis of TH in young adults and help physicians to prioritize intervention strategies for thymus rejuvenation in this population.

Keywords: thymus, hyperplasia, regeneration, lymphoma, chemotherapy, interleukin-7 receptor- $\alpha$, singlenucleotide polymorphisms 


\section{INTRODUCTION}

Atrophy of the thymus caused by cytotoxic drugs and glucocorticoid hormones remains a primary obstacle to full immune recovery following chemotherapy, which is dependent on high thymic output of new recent thymic emigrants (RTE) to replenish the naïve $\mathrm{T}$ cell pool $(1,2)$. Failure of restoration of thymus function may lead to increased risks of infections and tumor recurrence, particularly in elderly patients where the thymus is atrophied $(1,3)$. Enlargement of the thymus above baseline following chemotherapy, known as rebound thymic hyperplasia (TH), sometimes occurs during recovery from chemotherapy (4). TH may be related with robust thymic regeneration and is characterized by an increase in thymic size and density, concurrent with the restoration of thymic output of T cells (5-7). This phenomenon is common in children and adolescents and can occasionally be observed in young adults; however, it is rare in older patients (4, $8-11)$. Therefore, more effort should be made toward restoring thymic function in post-pubertal patients. However, the factors involved in thymus atrophy and regeneration are not fully understood, and approaches to stimulate rejuvenation of the thymus remain limited (12).

Clinical factors associated with thymic regrowth after chemotherapy have been explored in previous studies. Information on the occurrence of $\mathrm{TH}$ following chemotherapy obtained from different age groups revealed that the renewal ability of the thymus may be influenced by host age, and TH after chemotherapy is more common in younger populations with greater amounts of residual thymic tissues and higher thymic activity (4-11). Whereas, thymic regrowth is not likely associated with the tumor types and treatment. It can occur following treatment for various malignancies, of which malignant lymphoma is the most common (8). Besides, TH after cessation of chemotherapy does not appear to be influenced by the degree of lymphocyte depletion, but appears to be a common response to the withdrawal of chemotherapy $(4,6)$. There is also evidence that $\mathrm{TH}$ after chemotherapy maybe associated with endocrine dysfunctions (13). However, the factors mentioned above need to be further validated, since the knowledge of TH after chemotherapy was mainly obtained from analysis of small patient groups, and other potential factors related with $\mathrm{TH}$ have not been elicited.

Interleukin-7 (IL-7) acts as a non-redundant cytokine in thymic development (14). Under conditions of lymphopenia, it could optimize the long-term recovery of $\mathrm{T}$ cell diversity by restoring thymic function $(15,16)$. In patients with human immunodeficiency virus (HIV) infection, those with higher baseline levels of IL-7 had a higher incidence of TH after therapy (17). Patients with rheumatoid arthritis and type 1 diabetes demonstrating positive responses to treatment exhibited raised plasma IL-7 levels concurrent with renewed thymopoeisis $(18,19)$. Thus, IL-7 may be an important factor in thymic regeneration after injury. The responsiveness of IL-7 is dependent on the expression of the IL-7 receptor (IL-7R), which is a heterodimer consisting of the common $\gamma$-chain and the $\alpha$-chain (IL-7R $\alpha)(20,21)$. The gene encoding IL-7R $\alpha$ $(I L 7 R)$ is polymorphic, and single-nucleotide polymorphisms (SNPs) in $I L 7 R$ could potentially affect its transcription, thereby influencing IL-7R $\alpha$ expression levels and signal transduction (22-26). IL7R polymorphisms are likely to modulate the regulation, differentiation, and function of $\mathrm{T}$ cell subsets and are associated with the susceptibility to autoimmune diseases, the pathogenesis of graft-versus-host disease after hematopoietic stem cell transplantation (HSCT), and $\mathrm{T}$ cell repopulation after lymphocytopenia caused by HIV infection and HSCT (22-32). Moreover, SNPs in IL7R may influence thymic T cell development in patients with multiple sclerosis (MS) (25), indicating a possible role for these SNPs in the process of thymic regeneration after chemotherapy.

Considering these elements, the present study was aimed at examining clinical predictors for the occurrence of $\mathrm{TH}$ in a group of adult patients undergoing chemotherapy for lymphoma and exploring the possible contribution of $I L 7 R$ polymorphisms to thymic renewal capacity by detecting possible links between $I L 7 R$ SNPs and the recovery of thymic volume and output function after chemotherapy.

\section{MATERIALS AND METHODS}

\section{Patients}

Chinese Han patients with Hodgkin lymphoma (HL) and B cell lymphoma $(n=193)$ admitted to the Department of Hematology, the First Affiliated Hospital of Nanjing Medical University between January 2012 and June 2015 were included in this study. All subjects provided written informed consent in accordance with the requirements of the Declaration of Helsinki. The study was approved by the Ethics Committee of the First Affiliated Hospital of Nanjing Medical University. All patients accepted chemotherapy for lymphoma at diagnosis. For patients with HL, ABVD (adriamycin, bleomycin, vinblastine, and dacarbazine) regimen was administered. Patients with diffuse large $B$ cell lymphoma (DLBCL) were treated with R-CHOP (rituximab, cyclophosphamide, doxorubicin, vincristine, and prednisone) or R-DA-EPOCH (rituximab, etoposide, prednisone, vincristine, cyclophosphamide, and doxorubicin) regimen. Patients with follicular lymphoma (FL) or marginal zone lymphoma (MZL) accepted R-CHOP regimen mentioned above or R-COP (rituximab, cyclophosphamide, vincristine, and prednisone) regimen. Patients with Burkitt lymphoma (BL) received R-hyperCVAD (rituximab, cyclophosphamide, vincristine, doxorubicin, and dexamethasone) therapy alternating with R-MA (rituximab, methotrexate, and cytarabine). CT examinations were performed before (baseline), during (after three cycles of chemotherapy), and after $(0,3,6,9$, and 12 months post-chemotherapy) treatment. Simultaneously, blood samples were collected for flow cytometric analyses and peripheral blood mononuclear cells (PBMCs) separated by density gradient centrifugation. Patients $(n=28)$ who had no response to therapy, or had disease progression within 1 year after the end of treatment, were excluded. Patients $(n=15)$ who responded to chemotherapy and underwent HSCT as firstline consolidation were also excluded. For 16 patients, it was not possible to determine thymus size because of the growth of the lymphoma. The remaining 134 patients ( $82 \mathrm{DLBCL}, 15 \mathrm{HL}, 14$ FL, 10 MZL, 4 BL, and 9 others), aged 18-67 (median 41) years, were included. 


\section{Thymus Imaging}

Serial analyses of structural changes of the thymus were performed by reviewing CT images. Thymic size was scored using a thymic index on a scale from 0 to 5 as described elsewhere (6): 0 , no soft tissue; 1 , minimal soft tissue, barely recognizable; 2, minimal soft tissue, more obvious; 3 , moderate soft tissue; 4, moderate soft tissue of greater extent, almost mass-like; and 5, mass-like appearance, suggesting hyperplasia or thymoma. Thymic enlargement, or subsequent regression, was defined as a change in score of at least 1 on this $0-5$ scale. Enlargement of the thymus over baseline in the absence of any clinical, laboratory, or radiological signs of disease progression was interpreted as $\mathrm{TH}$.

\section{CD31+ RTE Detection}

For immunophenotypic analysis, whole blood samples were stained by four-color technique using anti-CD45RA-FITC, anti-CD31-PE, anti-CD4-PerCP, and anti-CD3-APC monoclonal antibodies and appropriate isotope controls (Becton Dickinson, USA). Erythrocytes were lysed with lysis solution (Becton Dickinson, USA). Lymphocyte data were analyzed by flow cytometry using a FACSCalibur (Becton Dickinson, USA). After gating for $\mathrm{CD}^{+}$and $\mathrm{CD}^{+}$cells, the percentage of $\mathrm{CD} 31^{+}$cells among all $\mathrm{CD} 45 \mathrm{RA}^{+} \mathrm{CD} 4^{+} \mathrm{T}$ cells was analyzed with CELLQuestTM software (Becton Dickinson, USA) as previously described (33). Whole blood lymphocyte counts were performed with an automated analyzer, and the absolute numbers of $\mathrm{CD} 31^{+}$ RTEs were determined by multiplying the total lymphocyte count by the percentage of $\mathrm{CD} 31^{+} \mathrm{CD} 45 \mathrm{RA}^{+} \mathrm{CD} 4^{+} \mathrm{T}$ cells.

\section{IL7R Genotyping}

Genomic DNA samples were extracted from PBMCs using a QIAamp DNA Blood Midi Kit (Qiagen, Germany), according to the manufacturer's instructions. On the basis of a literature search, four SNPs were chosen as our primary targets of investigation, including rs6897932 in exon 6, and rs7718919, rs11567685, and rs 11567686 in the promoter region, of $I L 7 R$ (22-25). Genotyping was performed by DNA sequencing. Briefly, the amplicons containing the promoter and exon 6 regions of $I L 7 R$ were PCRamplified from genomic DNA samples using primer sequences previously reported (22). PCR products were then purified by polyethylene glycol precipitation. Next, DNA sequencing was performed in both directions using the ABI Prism Big Dye Terminator version 3.1 sequencing kit and an ABI 3730XL Genetic Analyzer. Sequencing results were analyzed using Chromas 2.22 software (Technelysium, Australia).

\section{Single-Joint T-Cell Receptor Excision Circles (sjTREC) Analysis}

Serial quantification of sjTREC in the DNA of PBMCs was performed using a TaqMan real-time quantitative PCR assay and a StepOnePlus instrument (Applied Biosystems, USA), as previously described (5). A standard curve based on a plasmid preparation containing the sjTREC target sequence was plotted, and sjTREC values for samples were calculated using StepOne software (Applied Biosystems, USA). Samples were analyzed in triplicate, and median values calculated. Data are expressed as TRECs $/ 10^{6}$ cells.

\section{Statistics}

Continuous variables are expressed as means \pm SD and categorical variables as number of cases (percentage). Independent $t$ tests or Mann-Whitney $U$ tests were used to evaluate differences in numerical data. Chi-square or Fisher's exact tests were used to assess differences in categorical data and to compare genotype and allele frequencies between patients with and without $\mathrm{TH}$. Odds ratios (OR) and 95\% confidence intervals (CI) were calculated for the assessment of risk factors. Genotyping data were analyzed for Hardy-Weinberg equilibrium (HWE) and linkage disequilibrium (LD) using HaploView 4.2. LD blocks were identified using the CI setting. Univariate and multivariate logistic regression models were performed to investigate the associated factors for TH after chemotherapy. Variables with $P$ value $<0.10$ in the univariate analysis were selected for the multivariate analysis. The changes of thymic output at different time points were assessed by general linear models repeatedmeasure analysis using the statistical test within-subject contrasts. The effect of $I L 7 R$ SNPs on thymic output recovery was evaluated by general linear models repeated-measure analysis using between-subject contrasts. Data analysis was performed using SPSS2 1 statistical software. Values of $P<0.05$ were considered to be significant.

\section{RESULTS}

\section{The Occurrence of TH after Chemotherapy}

Thymic hyperplasia was observed in 38/134 (28.4\%) patients within intervals ranging from 1 to 10 months (median, 4 months) after the cessation of chemotherapy. Compared with those without TH $(n=96)$, patients with TH $(n=38)$ after chemotherapy were much younger, with larger thymic volumes, and higher thymic output before treatment $(P<0.05)$. Moreover, patients with $\mathrm{TH}$ were more commonly diagnosed with HL treated with chemotherapy only $(P<0.05)$. However, no significant differences were observed in sex, disease stage, and the pool size of peripheral $\mathrm{CD} 4^{+} \mathrm{T}$-cells at baseline between two groups $(P>0.05)$. The clinical characteristics of these patients are presented in Table 1.

\section{Recovery of Thymic Output after Chemotherapy}

The change in thymic output during and after chemotherapy was analyzed in 84 patients with available CD $31^{+}$RTE and sjTREC data at all follow-up time points (Figure 1). Consistent with our previous findings (5), both the numbers of CD $31^{+} \mathrm{RTE}$ and levels of sjTREC approached a nadir at the end of treatment $(P<0.001)$. During follow-up, CD31 ${ }^{+}$RTE counts and sjTREC levels rose significantly and reached pretreatment levels at 9 and 6 months after the end of chemotherapy, respectively $(P=0.035$ and 0.001 , respectively).

\section{The Effect of IL7R Polymorphisms on TH after Chemotherapy}

Genotypes for rs11567686 did not conform to $\operatorname{HWE}(P=0.029)$ and were excluded from further analyses. Genotypes of the three remaining SNPs were all consistent with HWE $(P>0.05)$; 
TABLE 1 | Baseline characteristics of patients with and without thymic hyperplasia (TH) after chemotherapy.

\begin{tabular}{|c|c|c|c|}
\hline Characteristics & Patients with TH $(n=38)$ & Patients without TH $(n=96)$ & $P$-value \\
\hline Age (years), median (IOR) & $30(18-53)$ & $48(18-67)$ & $<0.001$ \\
\hline \multicolumn{4}{|l|}{ Gender, n (\%) } \\
\hline Female & 20/38 (53) & $59 / 96(61)$ & \multirow[t]{2}{*}{0.436} \\
\hline Male & $18 / 38(47)$ & 37/96 (39) & \\
\hline \multicolumn{4}{|l|}{ Disease type, n (\%) } \\
\hline DLBCL & $18 / 38(47)$ & 64/96 (67) & \multirow[t]{3}{*}{0.038} \\
\hline $\mathrm{HL}$ & $8 / 38(21)$ & 7/96 (8) & \\
\hline Others & $12 / 38(32)$ & 25/96 (26) & \\
\hline \multicolumn{4}{|l|}{ Disease stage, n (\%) } \\
\hline$|-| \mid$ & $18 / 38(47)$ & 33/96 (34) & \multirow[t]{2}{*}{0.163} \\
\hline III-IV & 20/38 (53) & 63/96 (66) & \\
\hline \multicolumn{4}{|l|}{ Treatment, n (\%) } \\
\hline Chemotherapy & $8 / 38(21)$ & 7/96 (8) & \multirow[t]{2}{*}{0.023} \\
\hline Chemotherapy + rituximab & 30/38 (79) & 89/96 (92) & \\
\hline CD4 ${ }^{+} \mathrm{T}$ cells counts $\left(\times 10^{9} / \mathrm{L}\right)$ & $642.27 \pm 385.79$ & $577.67 \pm 365.14$ & 0.756 \\
\hline \multicolumn{4}{|l|}{ Thymic index, n (\%) } \\
\hline 0 & $3 / 38(8)$ & $45 / 96(47)$ & \multirow[t]{5}{*}{$<0.001$} \\
\hline 1 & $10 / 38(26)$ & 23/96 (24) & \\
\hline 2 & $19 / 38(50)$ & 21/96 (22) & \\
\hline 3 & $5 / 38(13)$ & 6/96 (6) & \\
\hline 4 & $1 / 38(3)$ & $1 / 96(1)$ & \\
\hline \multicolumn{4}{|l|}{ Thymic output } \\
\hline CD31+RTE (×109/L) & $194.85 \pm 158.31$ & $98.12 \pm 84.87$ & 0.036 \\
\hline sjTREC (copies/106 PBMCs) & $10,381.09 \pm 8,393.22$ & $5,109.70 \pm 7,162.70$ & 0.042 \\
\hline
\end{tabular}

DLBCL, diffuse large B cell lymphoma; HL, Hodgkin lymphoma; RTE, recent T cell emigrant; sjTREC, single-joint T-cell receptor rearrangement excision circle; PBMCs, peripheral blood mononuclear cells.
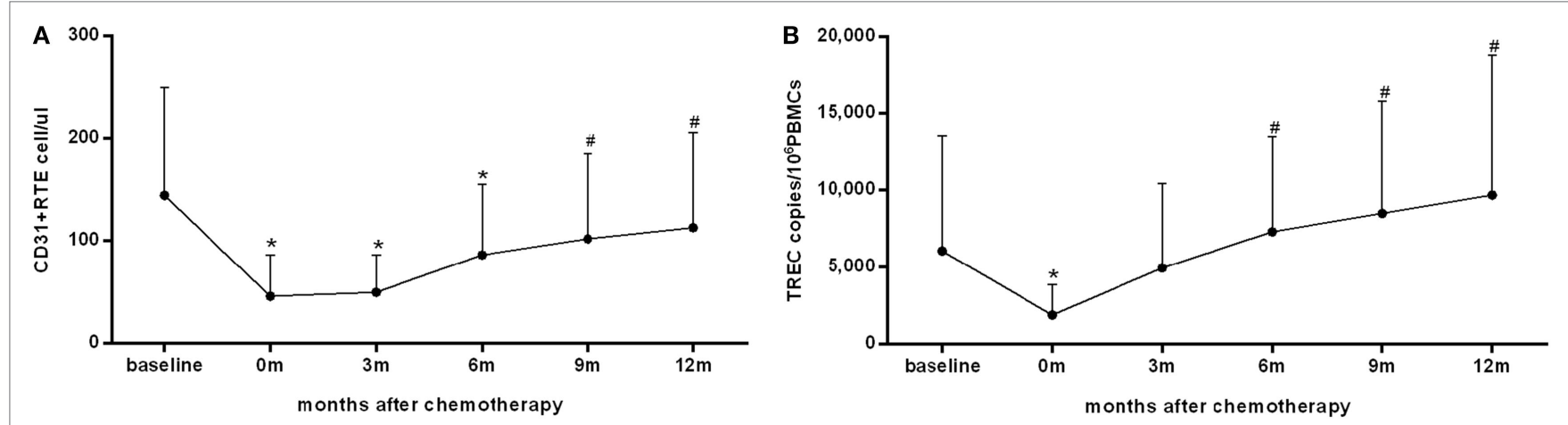

FIGURE 1 | The recovery of thymic output measured by CD31+ recent thymic emigrants (RTE) (A) and single-joint T-cell receptor excision circles (sjTREC) (B) in peripheral blood within $\mathbf{1}$ year after the end of chemotherapy in $\mathbf{8 4}$ patients. Both the CD31+ RTE counts and sjTREC levels decreased to the nadir at the end of treatment and recovered within 1 year of follow-up. Data are shown as means \pm SD. $P$ values were assessed by general linear model analysis for repeated-measure data. ${ }^{*} P<0.001$ vs. baseline levels; ${ }^{*} P<0.05$ vs. the nadir at the end of treatment.

however, the minor allele frequency of rs11567685 (0.019\%) was too low for differences between the two groups to be meaningfully analyzed. Therefore, allele and genotype frequencies for rs7718919 and rs6897932 in patients with and without TH were compared (Table 2).

The minor T allele of rs7718919 was significantly more frequent in patients with $\mathrm{TH}$ compared with those without $\mathrm{TH}(P=0.031$, $\mathrm{OR}=2.171,95 \%$ CI $1.064-4.433$, T vs. G). We also detected a significant difference under a recessive model with regard to the distribution of rs7718919 genotype frequencies between patients with and without $\mathrm{TH}(P=0.027, \mathrm{OR}=2.528,95 \% \mathrm{CI}$ 1.097-5.826, TT + GT vs. GG); however, no significant evidence was detected under a dominant model $(P=0.622$, OR $=1.722$, 95\% CI 0.276-10.737, TT vs. GG + GT). Moreover, no significant difference was found with respect to the distribution of allele and genotype frequencies of rs6897932 between subjects with and without TH $(P=0.383$ and 0.282 , respectively).

Due to high diversity and weak LD $\left(r^{2}=0.365\right)$ between rs7718919 and rs6897932, haplotype analyses were not possible in our study cohort. 
TABLE 2 | Genotype distribution and allele carriage rates of two single-nucleotide polymorphisms (SNPs) in the gene encoding IL-7R $\alpha$ in patients with and without thymic hyperplasia (TH) following chemotherapy.

\begin{tabular}{|c|c|c|c|c|c|}
\hline SNP locus & Genotype/allele & $\begin{array}{l}\text { Patients with } \\
\text { TH }(n=38)\end{array}$ & $\begin{array}{l}\text { Patients without } \\
\text { TH }(n=96)\end{array}$ & $\begin{array}{l}\text { Odds ratios } \\
{[95 \% \text { confidence intervals] }}\end{array}$ & $P$-value \\
\hline \multirow[t]{9}{*}{ rs7718919 } & GG & $24(0.632)$ & $78(0.812)$ & & 0.084 \\
\hline & GT & $12(0.316)$ & $15(0.156)$ & & \\
\hline & $\pi$ & $2(0.053)$ & $3(0.031)$ & & \\
\hline & $\pi$ & $2(0.053)$ & $3(0.031)$ & 1.722 [0.276-10.737] & 0.622 \\
\hline & $G G+G T$ & $36(0.947)$ & 93 (0.969) & & \\
\hline & $\mathrm{GT}+\mathrm{TT}$ & $14(0.368)$ & $18(0.187)$ & $2.528[1.097-5.826]$ & 0.027 \\
\hline & GG & $24(0.632)$ & $78(0.813)$ & & \\
\hline & $\mathrm{T}$ & $16(0.211)$ & $21(0.109)$ & $2.171[1.064-4.433]$ & 0.031 \\
\hline & $\mathrm{G}$ & $60(0.789)$ & $171(0.891)$ & & \\
\hline \multirow[t]{9}{*}{ rs6897932 } & $\mathrm{CC}$ & $27(0.711)$ & $59(0.615)$ & & 0.383 \\
\hline & CT & $11(0.289)$ & $34(0.354)$ & & \\
\hline & $\pi$ & $0(0.000)$ & $3(0.031)$ & & \\
\hline & $\mathrm{CC}+\mathrm{CT}$ & $38(1.000)$ & 93 (0.969) & 1.413 [1.265-1.578] & 0.577 \\
\hline & $\pi$ & $0(0.000)$ & $3(0.031)$ & & \\
\hline & $\mathrm{CC}$ & $27(0.711)$ & $59(0.615)$ & 1.539 [0.683-3.469] & 0.325 \\
\hline & $\mathrm{CT}+\pi$ & $11(0.289)$ & $37(0.385)$ & & \\
\hline & $\mathrm{C}$ & $65(0.855)$ & $152(0.792)$ & $1.555[0.751-3.220]$ & 0.282 \\
\hline & $\mathrm{T}$ & $11(0.145)$ & $40(0.208)$ & & \\
\hline
\end{tabular}

\section{The Effect of IL7R Polymorphisms on the Recovery of Thymic Output after Chemotherapy}

As previously shown in Ref. (4), thymic regeneration after chemotherapy manifests as an increase in thymic volume, concurrent with the restoration of thymopoiesis. We investigated the influence of rs7718919 and rs6897932 on the renewal of thymopoiesis following chemotherapy in 84 patients with thymic output data available for all follow-up time points.

The effect of rs7718919 genotypes was tested using a recessive model (TT + GT vs. GG), due to few cases carrying the minor allele T. By general linear models repeated-measure analysis, no impact of rs7718919 genotypes was found on the recovery of CD $31^{+}$RTEs counts and sjTREC levels within 1 year of follow-up $(P=0.743$ and 0.642 , respectively) (Figure S1 in Supplementary Material). Similarly, the effect of rs6897932 genotype was tested using a recessive model (CC vs. CT $+\mathrm{TT}$ ) and no impact was found on the restoration of thymic output measured by both CD $31^{+}$RTEs counts and sjTREC levels $(P=0.913$ and 0.896 , respectively) (Figure S2 in Supplementary Material). Baseline characteristics, including age, sex, disease type and stage, $\mathrm{CD} 4^{+}$ cell counts, and output function at baseline were comparable between the two groups with different genotypes $(P>0.05)$ (Table S1 in Supplementary Material).

\section{Analysis of Factors Associated with TH after Chemotherapy}

The univariate analysis results showed significant differences between patients with and without TH in terms of host age, disease type, pretreatment thymic size and CD31+ RTE counts, and rs7718919 genotype $(P<0.05)$ (Table 3$)$. Then, by multivariate analysis, it was concluded that host age under 30 is the only independent predictive factor for the occurrence of $\mathrm{TH}$ after chemotherapy $(P=0.002, \mathrm{OR}=4.710,95 \% \mathrm{CI}: 1.727-12.845)$. A higher probability of thymic regrowth following chemotherapy was observed in subjects with the T allele of rs7718919; however, this trend did not reach statistical significance $(P=0.093$, $\mathrm{OR}=2.308$, 95\%CI: 0.875-6.091) (Table 3).

\section{DISCUSSION}

Reactive TH after chemotherapy is a well-documented phenomenon in children, adolescents, and young adults, but is rare in older patients, suggesting a high thymic regenerative capacity in the young $(4,5,8-11)$. However, by monitoring thymic output, it was determined that substantial output is maintained into late adulthood despite the decline of thymic function with age, and even the aged thymus retains the ability to renew thymopoiesis (34). This study further explored the influence of age on the renewal capacity of the thymus following chemotherapy. In the cohort of adults accepting chemotherapy for lymphoma, $\mathrm{TH}$ was observed in $38(28.4 \%)$ cases aged 18-53 years (median 30 ), who were much younger, with larger thymic volumes and higher thymic output before treatment than those without $\mathrm{TH}$, suggesting a high renewal capacity was retained in the thymus of young adults. In contrast, $\mathrm{TH}$ was observed in none of the elderly, possibly due to a decreased regenerative capacity of the thymus with age, which could represent a decline in the intrinsic capacity of lymphopoietic stem cells and/or changes in the thymic microenvironment (7). Besides, by multivariate analysis, patient age under 30 was revealed as an independent predictor for the occurrence of $\mathrm{TH}$, further supporting the conclusion that thymic renewal capacity after chemotherapy is age-dependent. 
TABLE 3 | The univariate and multivariate analysis of factors influencing the occurrence of thymic hyperplasia after chemotherapy.

\begin{tabular}{|c|c|c|c|c|}
\hline Parameters & $\begin{array}{c}\text { Univariate analysis } \\
\text { Odds ratios (OR) } \\
\text { [95\% confidence intervals }(\mathrm{CI})]\end{array}$ & $P$-value & $\begin{array}{c}\text { Multivariate analysis } \\
\text { OR }[95 \% \mathrm{Cl}]\end{array}$ & $P$-value \\
\hline Age $\leq 30$ years & $8.054[3.415-18.991]$ & $<0.001$ & $4.710[1.727-12.845]$ & 0.002 \\
\hline Gender: female & $1.435[0.673-3.062]$ & 0.350 & & \\
\hline Disease type: HL & $3.390[1.134-10.140]$ & 0.029 & $2.279[0.646-8.036]$ & 0.200 \\
\hline Disease stage: |-|| & $1.718[0.801-3.687]$ & 0.165 & & \\
\hline Baseline thymic index $\geq 2$ & $4.670[2.059-10.412]$ & $<0.001$ & $2.087[0.774-5.628]$ & 0.146 \\
\hline Baseline CD4+ ${ }^{+}$cell counts $\geq 600 \times 10^{9} / \mathrm{L}$ & $1.950[0.445-8.548]$ & 0.376 & & \\
\hline Baseline CD31+ RTE counts $\geq 150 \times 10^{9} / \mathrm{L}$ & $3.896[1.059-14.326]$ & 0.041 & $2.160[0.430-10.845]$ & 0.350 \\
\hline Baseline sjTREC levels $\geq 7,000$ copies $/ 10^{6}$ PBMCs & $4.050[0.974-16.842]$ & 0.054 & $3.052[0.277-18.040]$ & 0.362 \\
\hline Rs7718919 genotype: TT + GT & $2.528[1.097-5.826]$ & 0.029 & $2.308[0.875-6.091]$ & 0.093 \\
\hline
\end{tabular}

HL, Hodgkin lymphoma; RTE, recent T cell emigrant; sjTREC, single-joint T-cell receptor rearrangement excision circle; PBMCs, peripheral blood mononuclear cells.

Consistent with this, we have revealed in an earlier study that, the majority (70\%) of $\mathrm{TH}$ following chemotherapy was found in individuals under 30 years, although it can occasionally be observed in middle-aged patients (10). Furthermore, the renewal of thymopoiesis following chemotherapy was evaluated in the present study. The fast restoration of thymic output within 1 year of follow-up observed in these adults suggested a renewed thymopoiesis. Thymus regeneration, manifesting as partial reversal of thymic atrophy concurrent with a renewed thymopoiesis, in young adults undergoing chemotherapy would contribute to immune recovery, enhancing the clinical relevance of developing strategies to booster thymic activity in this population $(2,5,12)$.

Clinical features except for patient age associated with thymic regrowth after chemotherapy have not been studied in-depth. Based on previous observations, it was seemed that, $\mathrm{TH}$ is neither tumor nor treatment specific $(4,6,8)$. This study revealed that, patients with $\mathrm{TH}$ after chemotherapy were more commonly diagnosed with HL than those without TH. While, by multivariate analysis, the diagnosis of HL was not associated with the occurrence of $\mathrm{TH}$ independently, it can be conceived that, the lower age of patients with HL may contribute to a higher probability of thymic regrowth. Whether thymic regrowth following chemotherapy is correlated with the biological properties of the tumors remains unknown. It should be reminded that, the influence of rituximab usage on the occurrence of TH post-chemotherapy cannot be excluded, since HL patients were treated with chemotherapy only, in contrast with patients with B cell lymphoma accepted chemotherapy combined with rituximab. Emerging data suggest that rituximab has an inhibitory effect on effector $\mathrm{T}$ cells and could induce regulatory $\mathrm{T}$ cells expansion, which may be related to $\mathrm{B}$ cell deprivation resulting in impaired antigen-presenting cells activity and reduced production of co-stimulatory and immuno-modulatory molecules taking part in $\mathrm{T}$ cell activation and interactions; besides, it could exert a direct effect on $\mathrm{T}$ cells by binding to a specific T cell subset expressing CD20 (35). A recent study confirmed that, CD20 was also expressed on mature medullary thymocytes (36). Therefore, rituximab may intervene T cell development in the thymus by targeting $\mathrm{CD} 20^{+}$thymocytes. Unfortunately, this study design precluded an analysis for the effect of rituximab.
Whether the use of rituximab could affect thymic activity and thus delay the regeneration of the thymus in patients with $\mathrm{B}$ cell lymphoma deserves further studies. Moreover, TH was demonstrated to be caused by chemotherapy-induced gonadal atrophy, which results in increased luteinizing hormone secretion (13), and sex steroid ablation has been regarded as an important candidate strategy for the improvement of thymic regeneration (12). Thus, the sex-related endocrine status is presumed to influence the renewal capacity of the thymus after chemotherapy. However, sex ratio was comparable in cases with and without TH in this study, excluding sex as a predictor for TH. Measurement of sex-related endocrine function is warranted to clarify this issue. In addition, the occurrence of $\mathrm{TH}$ in this cohort of adults we studied was not influenced by the size of peripheral $\mathrm{CD}^{+}{ }^{+} \mathrm{T}$ cell pool as expected, since renewed thymopoiesis correlated with $\mathrm{TH}$ play a limited role in the early repopulation of peripheral $\mathrm{CD} 4^{+} \mathrm{T}$ cells following chemotherapy in adults (10).

The mechanisms for the reconstitution of thymic cellularity, accompanied by the restoration of thymic $\mathrm{T}$ cell development, after the removal of chemo-therapeutic toxicity is complex $(37,38)$. Interleukin-7, a growth and anti-apoptotic factor for $\mathrm{T}$ cells, was found to be associated with thymus enlargement in HIV patients undergoing antiretroviral therapy, suggesting an important role of IL-7 in thymus regrowth (17). Consistent with this findings, administration of recombinant human IL-7 was shown to increase the numbers of RTEs and levels of TRECs and results in a broadening of the T-cell receptor repertoire, indicating increased thymopoiesis (39). The response of thymocytes to IL-7 correlates with IL-7R $\alpha$ expression. Accordingly, this study focused on four specific SNPs within the IL7R locus, known to influence the IL-7R $\alpha$ expression on T cells (23-25), and explored their potential contributions to the thymic regeneration after chemotherapy in adults with lymphoma. It was found that the frequencies of the minor allele T and the TT + GT genotype of rs7718919, located in the promoter region of $I L 7 R$, were higher in patients with TH compared with those without TH. These results provided a possible link between rs7718919 and thymus enlargement after chemotherapy. Considering rs7718919 could potentially influence the expression of IL-7R $\alpha$ as a transcription factor binding site (30), it is conceivable that it may affect 
IL-7-mediated regulation of thymocytes, and thus be implicated in thymic regrowth. However, this association between rs7718919 and the occurrence of $\mathrm{TH}$ was not confirmed by multivariate analysis. Moreover, we did not observe an accelerated recovery of thymic output after chemotherapy in patients with TT + GT genotype compared with GG genotype carriers, suggesting that the presence of the rs7718919 T allele had limited effects on the restoration of thymopoiesis. Consistent with these results, Shamim et al. (31) were unable to demonstrate any association between $I L 7 R$ polymorphisms and TREC levels before or after HSCT in a Danish cohort. Nevertheless, the influence of rs7718919 polymorphisms on the renewal of thymopoiesis should be carefully evaluated. As IL-7R $\alpha$ expression is finely tuned and differentially regulated during thymocyte development (21), it is important to better understand which thymocyte subset could be influenced by the altered IL-7 signaling associated with rs7718919 and to what extent this could affect thymic T cell development.

This study also investigated rs6897932, a missense polymorphism located in exon 6 of $I L 7 R$, which encodes the trans-membrane region of IL-7R $\alpha$. The $\mathrm{C}$ allele of rs6897932 is associated with increased skipping of exon 6 , resulting in a higher ratio of membrane-bound vs. soluble receptor, which could cause reduced IL-7R $\alpha$ expression on T cells (26). In a Danish cohort, homozygosity for the rs6897932 T-allele was associated with more rapid $\mathrm{CD} 4^{+} \mathrm{T}$-cell recovery after the initiation of antiretroviral therapy (28). Unfortunately, the design of that study did not allow evaluation of whether the increase in $\mathrm{CD} 4^{+} \mathrm{T}$ cells was caused by increased thymopoiesis or expansion of the peripheral T-cell pool. Our study revealed no significant association of rs6897932 polymorphisms with either thymic enlargement or the restoration of thymic output, indicating a limited role for rs6897932 variants in thymopoiesis recovery after chemotherapy. This is consistent with the report by Broux et al. (25) of no influence of rs6897932 polymorphisms on thymopoiesis, as measured by the frequency of $\mathrm{CD} 31^{+}$RTEs in MS patients and healthy controls. Further studies investigating the potential effects of rs6897932 on the expression of IL-7R $\alpha$ and its possible influence on the affinity and/or signaling of IL-7R $\alpha$ in various thymocyte subsets are warranted to elucidate its role on thymopoietic activity. In addition, rs11567685, located in the promoter region of $I L 7 R$, was included in this study. It could potentially influence thymic development and peripheral homeostasis of $\mathrm{T}$ cells by altering the expression of IL-7R $\alpha$ on $\mathrm{CD} 4^{+} \mathrm{T}$ cells in MS patients (25). Unfortunately, the effect of rs11567685 on the recovery of thymic cellularity and function after chemotherapy in this study cannot be evaluated, due to its low minor allele frequency.

This study has some limitations. Our observations were based on relatively few cases, and this may have masked significant effects due to lack of statistical power. In addition, thymic output measured by determination of sjTREC and CD $31^{+}$RTE may be influenced by the proliferation of peripheral $\mathrm{T}$ cells, potentially leading to an underestimation of thymic activity $(40,41)$. Hence, future studies with larger sample sizes and more accurate tools for thymic output measurement are necessary to further define the factors associated with thymic regrowth after chemotherapy. Moreover, evaluating the effect of rituximab usage on thymus regeneration following chemotherapy by comparing the occurrence of TH and the recovery of thymic output in two groups with comparable clinical features, including age, sex, disease type and stage, thymic size, and output function at baseline, is warranted in future studies.

To conclude, these findings suggest that patient age is an independent predictor for thymic regrowth after chemotherapy, which should promote awareness among physicians to make a timely diagnosis of $\mathrm{TH}$ in young adults and help physicians to prioritize intervention strategies for thymus rejuvenation after chemotherapy in this population. Besides, the potential link between the IL7R SNP and thymic regrowth after chemotherapy warrant further investigations, given the key roles of IL-7 and IL-7R $\alpha$ in thymic development. These results will contribute to an improved understanding of the mechanisms involved in thymic regeneration.

\section{ETHICS STATEMENT}

Ethics approval and consent to participate was obtained. In accordance with the 1975 guidelines of the Declaration of Helsinki and after obtaining approval for the study from the Ethics Committee of the First Affiliated Hospital of Nanjing Medical University, the medical staff of each participating center informed their patients of the potential risks and benefits of the planned treatment and the need for periodical clinical and laboratory checkups in detail. Informed consent was obtained from all the subjects involved in this study.

\section{AUTHOR CONTRIBUTIONS}

WX and J-YL conceived and designed the study; D-PS, LW, C-YD, and J-HL contributed to the experimental design and provided intellectual input; D-PS and Y-JW performed experiments; LW, $\mathrm{H}-\mathrm{YZ}$, and LF contributed patients and provided clinical data; D-PS and LW analyzed data and wrote the manuscript.

\section{FUNDING}

This study was supported by National Natural Science Foundation of China (81170485, 81170488, 81370657, and 81470328), Priority Academic Program Development of Jiangsu Higher Education Institute (JX10231801), Project of National Key Clinical Specialty, National Science \& Technology Pillar Program (2014BAI09B12), and a project funded by Jiangsu Provincial Special Program of Medical Science (BL2014086).

\section{SUPPLEMENTARY MATERIAL}

The Supplementary Material for this article can be found online at http://journal.frontiersin.org/article/10.3389/fimmu. 2016.00654/full\#supplementary-material. 


\section{REFERENCES}

1. Williams KM, Hakim FT, Gress RE. T cell immune reconstitution following lymphodepletion. Semin Immunol (2007) 19:318-30. doi:10.1016/j. smim.2007.10.004

2. Legrand N, Dontje W, van Lent AU, Spits H, Blom B. Human thymus regeneration and T cell reconstitution. Semin Immunol (2007) 19:280-8. doi:10.1016/j. smim.2007.10.001

3. Haynes BF, Sempowski GD, Wells AF, Hale LP. The human thymus during aging. Immunol Res (2000) 22:253-61. doi:10.1385/IR:22:2-3:253

4. Choyke PL, Zeman RK, Gootenberg JE, Greenberg JN, Hoffer F, Frank JA. Thymic atrophy and regrowth in response to chemotherapy: CT evaluation. AJR Am J Roentgenol (1987) 149:269-72. doi:10.2214/ajr.149.2.269

5. Sun DP, Jin H, Ding CY, Liang JH, Wang L, Fan L, et al. Thymic hyperplasia after chemotherapy in adults with mature B cell lymphoma and its influence on thymic output and CD4 T cells repopulation. Oncoimmunology (2016) 5:e1137417. doi:10.1080/2162402X.2015.1137417

6. Sfikakis PP, Gourgoulis GM, Moulopoulos LA, Kouvatseas G, Theofilopoulos AN, Dimopoulos MA. Age-related thymic activity in adults following chemotherapy-induced lymphopenia. Eur J Clin Invest (2005) 35:380-7. doi:10.1111/j.1365-2362.2005.01499.x

7. Mackall CL, Fleisher TA, Brown MR, Andrich MP, Chen CC, Feuerstein IM, et al. Age, thymopoiesis, and CD4+ T-lymphocyte regeneration after intensive chemotherapy. N Engl J Med (1995) 332:143-9. doi:10.1056/ NEJM199501193320303

8. Zhen Z, Sun X, Xia Y, Ling J, Cai Y, Wang J, et al. Clinical analysis of thymic regrowth following chemotherapy in children and adolescents with malignant lymphoma. Jpn J Clin Oncol (2010) 40:1128-34. doi:10.1093/jjco/hyq149

9. Yarom N, Zissin R, Apter S, Hertz M, Rahimi-Levene N, Gayer G. Rebound thymic enlargement on CT in adults. Int J Clin Pract (2007) 61:562-8. doi:10.1111/j.1742-1241.2006.00950.x

10. Sun DP, Ding CY, Wang L, Liang JH, Fan L, Wu YJ, et al. Thymic hyperplasia following chemotherapy in adults with lymphoma: F-fluorodeoxyglucose positron emission tomography/computed tomography findings and correlation with T cell repopulation. Leuk Lymphoma (2015) 56:2344-9. doi:10.3109/10 428194.2014.986480

11. Hara M, McAdams HP, Vredenburgh JJ, Herndon JE, Patz EF Jr. Thymic hyperplasia after high-dose chemotherapy and autologous stem cell transplantation. AJR Am J Roentgenol (1999) 173:1341-4. doi:10.2214/ajr.173.5.10541115

12. Heng TS, Chidgey AP, Boyd RL. Getting back at nature: understanding thymic development and overcoming its atrophy. Curr Opin Pharmacol (2010) 10:425-33. doi:10.1016/j.coph.2010.04.006

13. Sperandio P, Tomio P, Oliver RT, Fiorentino MV, Pagano F. Gonadal atrophy as a cause of thymic hyperplasia after chemotherapy. Br J Cancer (1996) 74:991-2. doi:10.1038/bjc.1996.474

14. von FU, Solvason N, Howard M, Murray R. The earliest T lineage-committed cells depend on IL-7 for Bcl-2 expression and normal cell cycle progression. Immunity (1997) 7:147-54. doi:10.1016/S1074-7613(00)80517-8

15. Mackall CL, Fry TJ, Bare C, Morgan P, Galbraith A, Gress RE. IL-7 increases both thymic-dependent and thymic-independent $\mathrm{T}$ cell regeneration after bone marrow transplantation. Blood (2001) 97:1491-7. doi:10.1182/blood. V97.5.1491

16. Alpdogan O, Schmaltz C, Muriglan SJ, Kappel BJ, Perales MA, Rotolo JA, et al. Administration of interleukin-7 after allogeneic bone marrow transplantation improves immune reconstitution without aggravating graft-versus-host disease. Blood (2001) 98:2256-65. doi:10.1182/blood.V98.7.2256

17. Ruiz-Mateos E, de la Rosa R, Franco JM, Martinez-Moya M, Rubio A, Soriano $\mathrm{N}$, et al. Endogenous IL-7 is associated with increased thymic volume in adult HIV-infected patients under highly active antiretroviral therapy. AIDS (2003) 17:947-54. doi:10.1097/00002030-200305020-00002

18. Ponchel F, Verburg RJ, Bingham SJ, Brown AK, Moore J, Protheroe A, et al. Interleukin-7 deficiency in rheumatoid arthritis: consequences for therapyinduced lymphopenia. Arthritis Res Ther (2005) 7:R80-92. doi:10.1186/ar1538

19. Hofer J, Hofer S, Zlamy M, Jeller V, Koppelstaetter C, Brandstätter A, et al. Elevated proportions of recent thymic emigrants in children and adolescents with type 1 diabetes. Rejuvenation Res (2009) 12:311-20. doi:10.1089/ rej.2009.0863

20. ElKassar N, Gress RE. An overview of IL-7 biology and its use in immunotherapy. J Immunotoxicol (2010) 7:1-7. doi:10.3109/15476910903453296
21. Carrette F, Surh CD. IL-7 signaling and CD127 receptor regulation in the control of T cell homeostasis. Semin Immunol (2012) 24:209-17. doi:10.1016/j. smim.2012.04.010

22. Teutsch SM, Booth DR, Bennetts BH, Heard RN, Stewart GJ. Identification of 11 novel and common single nucleotide polymorphisms in the interleukin-7 receptor-alpha gene and their associations with multiple sclerosis. Eur J Hum Genet (2003) 11:509-15. doi:10.1038/sj.ejhg.5200994

23. McKay FC, Swain LI, Schibeci SD, Rubio JP, Kilpatrick TJ, Heard RN, et al. Haplotypes of the interleukin 7 receptor alpha gene are correlated with altered expression in whole blood cells in multiple sclerosis. Genes Immun (2008) 9:1-6. doi:10.1038/sj.gene.6364436

24. Booth DR, Arthur AT, Teutsch SM, Bye C, Rubio J, Armati PJ, et al. Gene expression and genotyping studies implicate the interleukin 7 receptor in the pathogenesis of primary progressive multiple sclerosis. J Mol Med (Berl) (2005) 83:822-30. doi:10.1007/s00109-005-0684-y

25. Broux B, Hellings N, Venken K, Rummens JL, Hensen K, Van Wijmeersch B, et al. Haplotype 4 of the multiple sclerosis-associated IL-7Ra influences the frequency of RTE. Genes Immun (2010) 11:326-33. doi:10.1038/gene.2009.106

26. Gregory SG, Schmidt S, Puneet Seth P, Oksenberg JR, Hart J, Prokop A, et al. Interleukin 7 receptor alpha chain (IL7R) shows allelic and functional association with multiple sclerosis. Nat Genet (2007) 39:1083-91. doi:10.1038/ ng2103

27. Shamim Z, Spellman S, Haagenson M, Wang T, Lee SJ, Ryder LP, et al. Polymorphism in the interleukin-7 receptor-alpha and outcome after allogeneic hematopoietic cell transplantation with matched unrelated donor. Scand J Immunol (2013) 78:214-20. doi:10.1111/sji.12077

28. Hartling HJ, Thørner LW, Erikstrup C, Harritshøj LH, Kronborg G, Pedersen $\mathrm{C}$, et al. Polymorphism in interleukin-7 receptor a gene is associated with faster CD4+ T cell recovery after initiation of combination antiretroviral therapy. AIDS (2014) 28:1739-48. doi:10.1097/QAD.0000000000000354

29. Rajasuriar R, Booth DR, Gouillou M, Spelman T, James I, Solomon A, et al. The role of SNPs in the alpha-chain of the IL-7R gene in CD4+ T-cell recovery in HIV-infected African patients receiving suppressive cART. Genes Immun (2012) 13:83-93. doi:10.1038/gene.2011.65

30. Wang XS, Wen PF, Zhang M, Hu LF, Ni J, Qiu LJ, et al. Interleukin-7 receptor single nucleotide polymorphism rs6897932 (C/T) and the susceptibility to systemic lupus erythematosus. Inflammation (2014) 37:615-20. doi:10.1007/ s10753-013-9777-x

31. Shamim Z, Ryder LP, Christensen IJ, Toubert A, Norden J, Collin M, et al. Prognostic significance of interleukin-7 receptor- $\alpha$ gene polymorphisms in allogeneic stem-cell transplantation: a confirmatory study. Transplantation (2011) 91:731-6. doi:10.1097/TP.0b013e31820f08b2

32. O’Doherty C, Alloza I, Rooney M, Vandenbroeck K. IL7RA polymorphisms and chronic inflammatory arthropathies. Tissue Antigens (2009) 74:429-31. doi:10.1111/j.1399-0039.2009.01342.x

33. Hartling HJ, Gaardbo JC, Ronit A, Salem M, Laye M, Clausen MR, et al. Impaired thymic output in patients with chronic hepatitis $\mathrm{C}$ virus infection. Scand J Immunol (2013) 78:378-86. doi:10.1111/sji.12096

34. Jamieson BD, Douek DC, Killian S, Hultin LE, Scripture-Adams DD, Giorgi JV, et al. Generation of functional thymocytes in the human adult. Immunity (1999) 10:569-75. doi:10.1016/S1074-7613(00)80056-4

35. Avivi I, Stroopinsky D, Katz T. Anti-CD20 monoclonal antibodies: beyond B-cells. Blood Rev (2013) 27:217-23. doi:10.1016/j.blre.2013.07.002

36. Schuh E, Berer K, Mulazzani M, Feil K, Meinl I, Lahm H, et al. Features of human CD3+CD20+ T Cells. J Immunol (2016) 197:1111-7. doi:10.4049/ jimmunol.1600089

37. Nunes-Cabaco H, Sousa AE. Repairing thymic function. Curr Opin Organ Transplant (2013) 18:363-8. doi:10.1097/MOT.0b013e3283615df9

38. Boehm T, Swann JB. Thymus involution and regeneration: two sides of the same coin? Nat Rev Immunol (2013) 13:831-8. doi:10.1038/nri3534

39. Okamoto Y, Douek DC, McFarland RD, Koup RA. Effects of exogenous interleukin-7 on human thymus function. Blood (2002) 99:2851-8. doi:10.1182/ blood.V99.8.2851

40. Ferrando-Martınez S, Franco JM, Ruiz-Mateos E, Ordoñez A, Gutierrez E, Leal M. A reliable and simplified sj $\beta$-TREC ratio quantification method for human thymic output measurement. J Immunol Methods (2010) 352:111-7. doi:10.1016/j.jim.2009.11.007

41. Azevedo RI, Soares MV, Barata JT, Tendeiro R, Serra-Caetano A, Victorino RM, et al. IL-7 sustains CD31 expression in human naive CD4+ T cells and 
preferentially expands the CD31+ subset in a PI3K-dependent manner. Blood (2009) 113:2999-3007. doi:10.1182/blood-2008-07-166223

Conflict of Interest Statement: The authors declare that the research was conducted in the absence of any commercial or financial relationships that could be construed as a potential conflict of interest.
Copyright $\odot 2016$ Sun, Wang, Ding, Liang, Zhu, Wu, Fan, Li and Xu. This is an open-access article distributed under the terms of the Creative Commons Attribution License (CC BY). The use, distribution or reproduction in other forums is permitted, provided the original author(s) or licensor are credited and that the original publication in this journal is cited, in accordance with accepted academic practice. No use, distribution or reproduction is permitted which does not comply with these terms. 